\title{
Early Effects of a Hypocaloric, Mediterranean Diet on Laboratory Parameters in Obese Individuals
}

\author{
Marta Greco, ${ }^{1}$ Eusebio Chiefari, ${ }^{1}$ Tiziana Montalcini, ${ }^{2}$ Francesca Accattato, ${ }^{1}$ \\ Francesco S. Costanzo, ${ }^{3}$ Arturo Pujia, ${ }^{2}$ Daniela Foti, ${ }^{1}$ Antonio Brunetti, ${ }^{1}$ and Elio Gulletta ${ }^{1}$ \\ ${ }^{1}$ Department of Health Sciences, Magna Grecia University of Catanzaro, Viale Europa (Località Germaneto), 88100 Catanzaro, Italy \\ ${ }^{2}$ Department of Medical and Surgical Sciences, Magna Grecia University of Catanzaro, Viale Europa (Località Germaneto), \\ 88100 Catanzaro, Italy \\ ${ }^{3}$ Department of Clinical and Experimental Medicine, Magna Groecia University of Catanzaro, Viale Europa (Località Germaneto), \\ 88100 Catanzaro, Italy
}

Correspondence should be addressed to Daniela Foti; foti@unicz.it

Received 18 December 2013; Accepted 19 January 2014; Published 4 March 2014

Academic Editor: Massimiliano M. Corsi Romanelli

Copyright (C) 2014 Marta Greco et al. This is an open access article distributed under the Creative Commons Attribution License, which permits unrestricted use, distribution, and reproduction in any medium, provided the original work is properly cited.

Calorie restriction is a common strategy for weight loss in obese individuals. However, little is known about the impact of moderate hypocaloric diets on obesity-related laboratory parameters in a short-term period. Aim of this study was to evaluate the variation of laboratory biomarkers in obese individuals following a Mediterranean, hypocaloric (1400-1600 Kcal/die) diet. 23 obese, pharmacologically untreated patients were enrolled and subjected to the determination of anthropometric variables and blood collection at baseline, 1 and 4 months after diet initiation. After 4 months of calorie restriction, we observed a significant decrease in body weight and BMI (both $P<0.0001)$, insulin $(P=0.037)$, HOMA-IR $(P=0.026)$, leptin $(P=0.008)$, and $\mathrm{LDH}(P=0.023)$ and an increase in $\mathrm{EGF}(P=0.013)$. All these parameters, except LDH, varied significantly already at 1 month after diet initiation. Also, lower levels of insulin $(P=0.025)$, leptin $(P=0.023)$, and EGF $(P=0.035)$ were associated with a greater $(>5 \%)$ weight loss. Collectively, our data support a precocious improvement of insulin and leptin sensitivity after a modest calorie restriction and weight reduction. Moreover, EGF and LDH may represent novel markers of obesity, which deserve further investigations.

\section{Introduction}

Obesity, a chronic disorder whose prevalence is increasing in adults, adolescents, and children, is now considered a worldwide epidemic and an important issue in the health care $[1,2]$. In many populations, the prevalence of overweight and obesity has rapidly increased in the past 20 years. In the USA, more than $35 \%$ of adults and almost $17 \%$ of young people were classified as obese in 2009-2010 [3], and the lifetime risk of developing overweight or obesity was estimated to be roughly 50 and $25 \%$, respectively [4].

Obesity predisposes to a number of pathological conditions, including cardiovascular diseases $[5,6]$, type 2 diabetes $[7,8]$, metabolic syndrome [9], nonalcoholic fatty liver disease [10], certain types of cancer [11, 12], obstructive sleep apnea [13], osteoarthritis [14], and asthma [15], and increases the risk of premature death [16], leading to elevated health care costs worldwide [17].

Factors predisposing to obesity include excessive food intake, lack of physical activity, and genetic susceptibility [18]. However, obesity is a heterogeneous condition, and intraabdominal accumulation of fat tissue has been shown to have a higher impact on the development of cardiometabolic risk factors and related disorders than total excessive adiposity [19]. Increased free fatty acid availability, the release of proinflammatory cytokines and adipokines from adipose tissue, hepatic insulin resistance and inflammation, and the consequent dyslipidemia are among the many metabolic disorders associated with this condition. Hormonal status and drug treatments may also play an important role [20]. 
Also, increased levels of some hemostatic factors have been described that may explain the prothrombotic risk of obese patients [21].

Although surgical [22] and pharmacological therapies [23] have been shown to be useful, hypocaloric diet still represents the main and essential step in the treatment of obesity, together with increased physical activity and changes in lifestyle [24]. Weight loss, in fact, is an important goal and has proved to be beneficial in preventing health risks related to obesity $[25,26]$. In this context, many hypocaloric diets are available that can be potentially recommended, including Mediterranean diet [27].

The association between the effects of a low-calorie diet and obesity is however very complex and not yet fully understood. In particular, little is known about the pathogenetic mechanisms and their putative markers that link potential benefits of weight loss, in a short-term period, to the asymptomatic, obese patients.

To elucidate possible relationships between obesity, diet, and weight loss, herein, we evaluated the variation, at short time intervals, of some anthropometric and laboratory parameters in obese individuals, subjected to a Mediterranean, low-calorie diet. In the effort to identify useful precocious markers in the follow-up of these patients, we analysed laboratory parameters correlated with the pathophysiological changes linked to obesity, including indexes of insulin resistance, proinflammatory and prothrombotic markers, adipocytokines, and lipid profile.

\section{Materials and Methods}

2.1. Patients and Study Design. We designed a prospective, descriptive study in the routine management of patients with obesity.

52 patients that attended the Clinical Nutrition Operative Unit of our University and were not taking any medications were initially enrolled from January 2012 to May 2013. Due to the high drop-off rate, 23 patients (17 females and 6 males), age 52 (46-61 yrs) and average body mass index (BMI) $37 \mathrm{~kg} / \mathrm{m}^{2}$, completed the study. Obesity was classified, according to the WHO guidelines, in grade I (BMI: 30$34.9 \mathrm{~kg} / \mathrm{m}^{2}$ ), grade II (BMI: $35-39.9 \mathrm{~kg} / \mathrm{m}^{2}$ ), and severe obesity (BMI: $\geq 40 \mathrm{~kg} / \mathrm{m}^{2}$ ). Percentage of fat mass and percentage of lean mass were established at the enrollment by bioelectrical impedance analysis using the application Bodygram Pro (Akern, Pontassieve, FI, Italy). Any pharmacological treatment or intercurrent diseases during follow-up were assumed as exclusion criteria. Informed consent was obtained by each patient enrolled in the study.

After careful consideration of global nutrition, a personalized, Mediterranean hypocaloric diet (1400-1600 kcal/die) was prescribed, aiming at the achievement of a moderate weight loss. The proposed food plan included carbohydrates (55\% of total calories), proteins (20\% of total calories), and mostly mono- and polyunsaturated fats $(25 \%$ of total calories). The intake of cholesterol in the diet accounted for less than $300 \mathrm{mg} /$ day, while fibers were equal to 25-30 g/day. The diet was also balanced in terms of micronutrients intake.
Patients underwent two sessions of behavioral dietary counseling: one at the first visit, which focused on notions of nutrition, dietary errors, suggestions on food preparation techniques, and the importance of associating physical exercise (30 minutes of speed walking, 3 days/week) with diet therapy, and a second session at 1 month from dietary treatment, to reinforce adherence to diet. During the first visit, patients were also subjected to anthropometric determinations (body weight and height). During the first visit, patients were also subjected to anthropometric determinations, including body weight and height for BMI calculation, and blood collection. Fasting blood samples were collected from all participants with no caloric intake for at least $12 \mathrm{~h}$, and the following biochemical analyses were performed: glucose, insulin, cystatin C, adipokines (leptin, adiponectin, resistin, and visfatin), prothrombin time (PT), activated partial thromboplastin time (aPTT), fibrinogen, plasminogen activator inhibitor-1 (PAI-1), triglycerides, total cholesterol, and HDL cholesterol, $\gamma$-glutamyltransferase ( $\gamma \mathrm{GT})$, aspartate aminotransferase (AST), alanine aminotransferase (ALT), lactate dehydrogenase (LDH), highsensitivity $\mathrm{C}$ reactive protein (hsCRP), tumor necrosis factor $\alpha(\mathrm{TNF} \alpha)$, interleukins (IL): IL- $1 \alpha$, IL- $1 \beta$, IL- 2, IL- 4, IL-6, IL-8, and IL-10, monocyte chemoattractant protein-1 (MCP1 ), vascular endothelial growth factor (VEGF), and epidermal growth factor (EGF). Insulin resistance was calculated with the homeostatic model assessment method of insulin resistance (HOMA-IR) [28]. The same patients were visited and subjected to the same blood determinations after 1 and 4 months from dietary treatment. We established a cut-off of $5 \%$ at 4 months to indicate the efficacy of treatment.

2.2. Blood Sample Collection and Routine Work-Up. Blood was collected, after $12-14 \mathrm{~h}$ fasting, by antecubital venous puncture. Serum or plasma samples were obtained by centrifugation. Routine analyses, including blood count, PT, PTT and fibrinogen, fasting glucose, total cholesterol, HDL and LDL, triglycerides, AST, ALT, $\gamma \mathrm{GT}$, and LDH, were obtained from fresh samples, whereas aliquots of serum or citrated plasma were frozen at $-80^{\circ}$ for subsequent laboratory determinations (insulin, adipokines, cytokines, hsCRP, cystatin C, and PAI-1).

Blood count analysis was performed using ADVIA 2120 (Siemens, USA); PT, aPTT, and fibrinogen were performed on BCS XP (Siemens, USA); blood glucose, total cholesterol, HDL and LDL, triglycerides, AST, ALT, $\gamma$ GT, and LDH were performed on Cobas 6000 (Roche, Switzerland). All the above-mentioned assays were carried out according to the manufacturer's instructions.

Reference values of each measured analyte are reported in the supplementary table (see Supplementary Material available online at http://dx.doi.org/10.1155/2014/750860).

2.3. Measurements of Serum Cystatin $C$ and hsCRP. The determinations of serum concentrations of cystatin $C$ and hsCRP were carried out using a nephelometer BNII (Siemens, USA) by immunonephelometric methods, according to 
the manufacturer's instructions (Penia N Latex Cystatin C kit and CardioPhase hsCRP kit, Siemens, USA, resp.).

2.4. Measurement of Serum Cytokines. The serum concentrations of 12 different cytokines and growth factors (IL$1 \alpha$, IL-1 $\beta$, IL-2, IL-4, IL-6, IL-8, IL-10, IFN $\gamma$, TNF $\alpha$, MCP-1, VEGF, and EGF) were simultaneously determined using the biochip analyser Evidence Investigator (Randox Labs, UK) and the "Cytokine Array I and High sensitivity" kit, Randox, UK, according to the manufacturer's instructions. Briefly, the principle of this multianalyte testing relies on a sandwich ELISA, in which the analytes of interest are captured by specific antibodies bound to discrete regions of the surface chemistry of the biochip; horseradish peroxidase (HRP) labeled secondary antibodies, which specifically recognize the analytes, trigger a luminol-based electrochemiluminescent signal emission, registered by a CCD camera and quantified by a software.

2.5. Measurement of Serum Insulin and Adipokines. The serum concentration of insulin was measured by the ADVIA Centaur Immunoassay system (Siemens, USA), using a chemiluminescent immunoassay. Serum adipokines were determined by sandwich ELISA assays on the automated ELISA analyser Triturus (Grifols, USA), with the kits produced by Mediagnost, Germany (Adiponectin E09, sensitive leptin E077, resistin E50). Visfatin was determined by Visfatin C-Terminal Human, EIA Kit (Phoenix).

2.6. Statistical Analysis. Each quantitative trait was tested for normality using the Shapiro-Wilk normality test and, when required, it has been log-transformed. Any continuous variable was indicated as median and interquartile range. The nonparametric Friedman and Wilcoxon tests were used to evaluate the differences between the intragroup and anthropometric variables and laboratory parameters during the time of the study. A significance level of $P<0.05$ was set for a type I error in all analyses. Multivariate analysis was performed by incorporating age and sex as covariates using the linear regression procedure. All statistical procedures were performed by using the SPSS 20.0 software for Mac.

\section{Results}

The characteristics of the population studied are summarized in Table 1. Most participants (74\%) were women, and the mean age was 52 years. At baseline, the population enrolled was affected by grade I, grade II, and severe obesity $(34.8 \%$, $43.5 \%, 21.7 \%$, resp.), and the mean BMI was $37 \mathrm{~kg} / \mathrm{m}^{2}$. Data on body composition indicate the contribution of adiposity to body weight. Comparison of all the parameters examined at time of 0,1 , and 4 months from diet treatment is shown in Table 2. During the follow-up, body weight and BMI significantly decreased $(P<0.001)$ after calorie restriction, with an average reduction of body weight of $3.4 \%$ at 1 month and $5.8 \%$ at 4 months after dieting. After the 4 -month dietary intervention, in fact, body weight loss redistributed the status of patients and their proportions, as overweight (17.4\%)
TABLE 1: Demographic and anthropometric features of obese patients at the enrollment.

\begin{tabular}{lc}
\hline & Patients \\
\hline Race & Caucasian \\
$N$ & 23 \\
Female & 17 \\
Age $(\mathrm{yrs})$ & $52(46-61)$ \\
Height $(\mathrm{cm})$ & $155(153-167)$ \\
BMI $\left(\mathrm{Kg} / \mathrm{m}^{2}\right)$ & $37(32-39)$ \\
FM $(\%)$ & $43.5(34.5-46.0)$ \\
LM $(\%)$ & $37.0(33.0-43.7)$ \\
FM/LM & $1.2(0.8-1.4)$ \\
\hline
\end{tabular}

FM: fat mass; LM: lean mass, as calculated by bioelectrical impedance analysis. Continuous variables are expressed as median and quartiles.

and grade I (34.8\%) and grade II (26.1\%) obese, while the percentage of patients with severe obesity had no variation $(21.7 \%)$.

Weight loss led to an improvement in insulin sensitivity, as indicated by a decrement of both insulin $(P=0.037)$ and HOMA-IR index $(P=0.026)$. LDH showed a decrement after 4 months of treatment $(P=0.023)$, whereas leptin was the biomarker that varied more significantly throughout the whole time period $(P=0.008)$. Also, to note, EGF showed a significant increase $(P=0.013)$. Some other analytes showed an interesting trend of variability. In particular, a decremental trend has been observed for IL-6 and VEGF and for prothrombotic risk parameters, such as fibrinogen and PAI-1 (Table 2). To strengthen the precocious variation of the parameters examined, we compared all the variables after only one month of dietary intervention. Statistical analysis showed significant variations of insulin, HOMA-IR, leptin, resistin, EGF, and total cholesterol, confirming leptin as the most sensitive marker (Table 3 ).

Then, we analyzed the effect of weight loss on biochemical parameters after 4 months, compared subjects who obtained a decrease in their body weight greater than $5 \%$ with those who did not, and arbitrarily used a weight loss $>5 \%$ as a group variable. Notably, we observed a more significant variation of insulin $(P=0.025)$, leptin $(P=0.023)$, and EGF $(P=0.035)$ in patients achieving a greater body weight loss (Table 4$)$.

\section{Discussion}

Diet represents the most widespread tool to reduce weight in the obese individuals [29]. It has been shown that even a moderate weight loss of about $10 \%$ or less contributed to several health advantages that include improvement in metabolic parameters, reduction of blood pressure, and increase in longevity $[25,26]$. Also, there are circumstantial lines of evidence that calorie restriction improves insulin sensitivity and reduces systemic inflammation [30-33].

Several reports [27, 34-36] have emphasized the benefits of weight loss in obese individuals by evaluating physical and biochemical markers after at least 6 months from diet introduction, whereas little is known about possible earlier 
TABLE 2: Comparison of demographic, anthropometric, clinical, and biochemical features of obese patients at baseline and during follow-up.

\begin{tabular}{|c|c|c|c|c|}
\hline & Baseline & 1 month & 4 months & $P$ \\
\hline Weight (Kg) & $91(82-112)$ & $87(83-101)$ & $87(78-98)$ & $<0.001$ \\
\hline $\operatorname{BMI}\left(\mathrm{Kg} / \mathrm{m}^{2}\right)$ & $37(32-39)$ & $36(31-38)$ & $35(31-38)$ & $<0.001$ \\
\hline Total cholesterol (mg/dL) & $199(172-225)$ & $190(151-222)$ & $209(168-218)$ & 0.249 \\
\hline $\mathrm{HDL}(\mathrm{mg} / \mathrm{dL})$ & $50(38-57)$ & $44(39-53)$ & $49(41-57)$ & 0.073 \\
\hline $\mathrm{LDL}$ (mg/dL) & $131(95.0-160.0)$ & $117(95.0-153.0)$ & $120(95.0-149)$ & 0.444 \\
\hline Triglycerides (mg/dL) & $105(66-169)$ & $111(76-132)$ & $95(83-149)$ & 0.420 \\
\hline Fasting glucose (mg/dL) & 97 (91-107) & $96(91-99)$ & $94(87-104)$ & 0.168 \\
\hline Basal insulin $(\mu \mathrm{U} / \mathrm{mL})$ & $18.0(13.0-27.0)$ & $15.0(10.0-20.0)$ & $14.0(10.0-28.0)$ & 0.037 \\
\hline HOMA-IR & $4.0(3.0-6.0)$ & $4.0(2.0-5.0)$ & $3.0(3.0-6.0)$ & 0.026 \\
\hline$\gamma \mathrm{GT}(\mathrm{U} / \mathrm{L})$ & $22.0(15.0-44.0)$ & $21.0(14.0-40.0)$ & $19.0(13.0-65.0)$ & 0.299 \\
\hline AST (U/L) & $23.0(17.0-27.0)$ & $21.0(18.0-24.0)$ & $19.0(16.0-24.0)$ & 0.238 \\
\hline $\operatorname{ALT}(\mathrm{U} / \mathrm{L})$ & $27.0(19.0-34.0)$ & $25.0(18.0-35.0)$ & $22.0(17.0-33.0)$ & 0.220 \\
\hline $\mathrm{LDH}(\mathrm{U} / \mathrm{L})$ & $345(326-397)$ & $350.0(329-403)$ & $323(296-376)$ & 0.023 \\
\hline Adiponectin $(\mu \mathrm{g} / \mathrm{mL})$ & $5.0(3.0-7.0)$ & $4.0(3.0-6.0)$ & $5.0(3.0-7.0)$ & 0.923 \\
\hline Leptin $(\mathrm{ng} / \mathrm{mL})$ & $47.0(31.0-70.0)$ & $35.0(22.0-46.0)$ & $36.0(27.0-44.0)$ & 0.008 \\
\hline Resistin (ng/mL) & $5.0(4.0-6.0)$ & $5.0(5.0-7.0)$ & $5.0(4.0-7.0)$ & 0.267 \\
\hline Visfatin (ng/mL) & $7.0(5.0-8.0)$ & $7.0(6.0-9.0)$ & $7.0(6.0-9.0)$ & 0.559 \\
\hline Cystatin C (mg/dL) & $0.79(0.71-0.85)$ & $0.78(0.67-0.85)$ & $0.78(0.72-0.85)$ & 0.640 \\
\hline PT (\%) & $102(98-104)$ & $103(96-104)$ & 104 (99-110) & 0.211 \\
\hline aPTT (sec) & $27(26-28)$ & $28(27-30)$ & $27(25-29)$ & 0.001 \\
\hline Fibrinogen $(\mathrm{mg} / \mathrm{dL})$ & $325(274-369)$ & $324(287-349)$ & $308(260-341)$ & 0.676 \\
\hline PAI-1 (U/mL) & $5.0(2.0-6.0)$ & $3.0(1.0-6.0)$ & $3.0(1.0-4.0)$ & 0.610 \\
\hline hsCRP (mg/dL) & $2.8(1.8-7.0)$ & $2.8(1.61-5.2)$ & $4.0(1.2-6.4)$ & 0.568 \\
\hline IL-2 (pg/mL) & $0.0(0.0-5.0)$ & $3.0(0.0-5.0)$ & $3.0(0.0-6.0)$ & 0.154 \\
\hline $\mathrm{IL}-4(\mathrm{pg} / \mathrm{mL})$ & $0.0(0.0-2.0)$ & $0.0(0.0-2.0)$ & $0.0(0.0-0.0)$ & 0.409 \\
\hline IL-6 (pg/mL) & $1.3(1.0-2.0)$ & $1.3(1.0-2.0)$ & $1.0(1.0-2.0)$ & 0.394 \\
\hline IL-8 (pg/mL) & $10.0(7.0-28.0)$ & $14.0(6.0-38.0)$ & $14.0(10.0-25.0)$ & 0.535 \\
\hline IL-10 (pg/mL) & 0.0 & 0.0 & 0.0 & - \\
\hline VEGF (pg/mL) & $193(98-361)$ & $178(101-283)$ & $174(90-267)$ & 0.568 \\
\hline IFN $\gamma(\mathrm{pg} / \mathrm{mL})$ & $2.0(0.0-4.0)$ & $2.0(0.0-4.0)$ & $3.0(0.0-6.0)$ & 0.486 \\
\hline $\mathrm{TNF} \alpha(\mathrm{pg} / \mathrm{mL})$ & $3.0(3.0-5.0)$ & $3.0(3.0-6.0)$ & $4.0(3.0-7.0)$ & 0.867 \\
\hline $\mathrm{IL}-1 \alpha(\mathrm{pg} / \mathrm{mL})$ & 0.0 & 0.0 & 0.0 & - \\
\hline IL-1 $\beta(\mathrm{pg} / \mathrm{mL})$ & $0.8 \pm 2.0^{*}$ & $0.7 \pm 2.3^{*}$ & $0.8 \pm 2.7^{*}$ & 0.662 \\
\hline MCP-1 (pg/mL) & $351(206-395)$ & $352(232-515)$ & $367(214-485)$ & 0.302 \\
\hline $\mathrm{EGF}(\mathrm{pg} / \mathrm{mL})$ & $86(68-130)$ & $114(84-144)$ & $97(68-139)$ & 0.013 \\
\hline
\end{tabular}

Nonparametric Friedman test has been used for comparisons at three times. All variables are expressed as median and quartiles. ${ }^{*}$ These data are expressed as average \pm SD.

TABLE 3: Significant variations of anthropometric, clinical, and biochemical features of obese patients after one month of calorie restriction.

\begin{tabular}{lccc}
\hline & Baseline & 1 month & $P$ \\
\hline Weight $(\mathrm{Kg})$ & $91(82-112)$ & $87(83-101)$ & $<0.001$ \\
BMI $\left(\mathrm{Kg} / \mathrm{m}^{2}\right)$ & $37(32-39)$ & $36(31-38)$ & $<0.001$ \\
Basal insulin $(\mu \mathrm{U} / \mathrm{mL})$ & $18.0(13.0-27.0)$ & $15.0(10.0-20.0)$ & 0.011 \\
HOMA-IR & $4.0(3.0-6.0)$ & $4.0(2.0-5.0)$ & 0.006 \\
Leptin $(\mathrm{ng} / \mathrm{mL})$ & $46.0(31.0-70.0)$ & $35.0(22.0-46.0)$ & $<0.001$ \\
Resistin $(\mathrm{ng} / \mathrm{mL})$ & $5.0(4.0-6.0)$ & $5.0(5.0-7.0)$ & 0.017 \\
Total cholesterol $(\mathrm{mg} / \mathrm{dL})$ & $199(172-225)$ & $190(148-220)$ & 0.047 \\
EGF $(\mathrm{pg} / \mathrm{mL})$ & $86(68-130)$ & $114(84-144)$ & 0.018 \\
\hline
\end{tabular}

Nonparametric Wilcoxon test has been used for comparisons at two times. All variables are expressed as median and quartiles. 
TABLE 4: Effect of weight loss on biochemical parameters.

\begin{tabular}{|c|c|c|c|c|c|}
\hline & Weight loss $>5 \%$ & Weight loss $\leq 5 \%$ & Beta & $t$ & $P$ \\
\hline Total cholesterol (mg/dL) & $206(184-226)$ & $177(146-218)$ & 0.338 & 1.699 & 0.106 \\
\hline HDL (mg/dL) & $52(44-68)$ & $49(45-54)$ & 0.075 & 0.321 & 0.752 \\
\hline Triglycerides (mg/dL) & $89(79-154)$ & $101(71-134)$ & 0.020 & 0.081 & 0.936 \\
\hline Fasting glucose (mg/dL) & $95(88-119)$ & $94(86-103)$ & 0.300 & 1.181 & 0.252 \\
\hline Basal insulin $(\mu \mathrm{U} / \mathrm{mL})$ & $10.0(8.7-15.7)$ & $24.0(12.0-28.5)$ & -0.506 & -2.430 & 0.025 \\
\hline HOMA-IR & $3.0(2.0-3.2)$ & $5.0(3.0-6.0)$ & -0.435 & -2.040 & 0.056 \\
\hline$\gamma \mathrm{GT}(\mathrm{U} / \mathrm{L})$ & $19.5(14.2-56.0)$ & $17.0(11.0-81.0)$ & 0.123 & 0.487 & 0.632 \\
\hline AST (U/L) & $20.5(15.7-24.2)$ & $18.0(16.0-24.0)$ & 0.144 & 0.646 & 0.526 \\
\hline $\operatorname{ALT}(\mathrm{U} / \mathrm{L})$ & $19.0(17.2-32.0)$ & $23.0(14.5-33.5)$ & 0.329 & 1.317 & 0.203 \\
\hline $\mathrm{LDH}(\mathrm{U} / \mathrm{L})$ & $323(298-389)$ & $334(295-363)$ & -0.141 & -0.587 & 0.564 \\
\hline Adiponectin $(\mu \mathrm{g} / \mathrm{mL})$ & $6.0(4.0-7.25)$ & $4.0(3.0-5.0)$ & 0.298 & 1.164 & 0.259 \\
\hline Leptin $(\mathrm{ng} / \mathrm{mL})$ & $31.5(19-40.0)$ & $41.0(31.5-53.5)$ & -0.575 & -2.478 & 0.023 \\
\hline Resistin (ng/mL) & $5.0(4.0-8.0)$ & $6.0(4.0-9.0)$ & -0.258 & -0.940 & 0.359 \\
\hline Visfatin (ng/mL) & $7.5(6.0-10.0)$ & $7.0(6.0-8.5)$ & 0.088 & 0.353 & 0.729 \\
\hline Cystatin C (mg/dL) & $1.0(1.0-1.0)$ & $1.0(1.0-1.0)$ & - & - & - \\
\hline PT (\%) & $105(102-111)$ & $103(97-108)$ & 0.079 & 0.321 & 0.752 \\
\hline aPTT (sec) & $27(25-28)$ & $27(25-29)$ & -0.121 & -0.554 & 0.586 \\
\hline Fibrinogen (mg/dL) & $297(261-346)$ & $319(244-339)$ & -0.068 & -0.271 & 0.790 \\
\hline PAI-1 (U/mL) & $2.5(1.0-4.5)$ & $3.0(1.5-4.0)$ & 0.010 & 0.038 & 0.970 \\
\hline hsCRP (mg/dL) & $4.5(1.0-9.2)$ & $4.0(2.0-5.5)$ & 0.014 & 0.065 & 0.949 \\
\hline IL-2 (pg/mL) & $3.5(0.0-5.2)$ & $3.0(0.0-6.0)$ & 0.001 & 0.002 & 0.999 \\
\hline IL-4 (pg/mL) & $0.0(0.0-2.0)$ & $0.0(0.0-2.0)$ & - & - & - \\
\hline IL-6 (pg/mL) & $1.0(1.0-2.0)$ & $1.0(1.0-2.0)$ & 0.058 & 0.256 & 0.801 \\
\hline IL-8 (pg/mL) & $15.0(12.5-28.2)$ & $12.0(9.0-23.0)$ & 0.070 & 0.298 & 0.769 \\
\hline IL-10 (pg/mL) & 0.0 & 0.0 & - & - & - \\
\hline VEGF (pg/mL) & $230(69-347)$ & $157(100-238)$ & -0.025 & -0.105 & 0.917 \\
\hline $\mathrm{IFN} \gamma(\mathrm{pg} / \mathrm{mL})$ & $4.0(0.7-9.7)$ & $2.0(0.0-4.0)$ & 0.318 & 1.159 & 0.271 \\
\hline $\mathrm{TNF} \alpha(\mathrm{pg} / \mathrm{mL})$ & $4.0(2.7-7.2)$ & $5.0(4.0-7.5)$ & -0.191 & -0.887 & 0.386 \\
\hline IL-1 $\alpha(\mathrm{pg} / \mathrm{mL})$ & 0.0 & 0.0 & - & - & - \\
\hline $\mathrm{IL}-1 \beta(\mathrm{pg} / \mathrm{mL})$ & $1.4 \pm 4.2^{*}$ & $0.3 \pm 0.7^{*}$ & - & - & - \\
\hline MCP-1 (pg/mL) & $372(228-547)$ & $338(203-444)$ & 0.243 & 1.111 & 0.280 \\
\hline $\mathrm{EGF}(\mathrm{pg} / \mathrm{mL})$ & $77(54.5-106.2)$ & $121(87-156)$ & -0.468 & -2.273 & 0.035 \\
\hline
\end{tabular}

Multivariate linear regression analysis has been adjusted for gender and age. All variables are expressed as median and quartiles. ${ }^{*}$ These data are expressed as average \pm SD.

changes of these indexes. In particular, by comparing different hypocaloric diets, the Mediterranean diet has proved to be associated with a greater improvement of insulin sensitivity parameters [27]. The innovative aspects of our study consisted in the short-term assessment, in obese subjects, of clinical and laboratory parameters after a hypocaloric, Mediterranean diet and the achievement of a generally modest weight loss.

In our study, the efficacy of dietary treatment was proved by an average reduction of body weight of $3.4 \%$ at 1 month and $5.8 \%$ at 4 months and by a statistically significant reduction in BMI. Most studies conducted so far have considered a minimum of $5 \%$ body weight loss indispensable to obtain beneficial effects $[25,26,37]$. Interestingly, our findings show that, even after a smaller decrement of body weight, diet is associated with a precocious, statistically significant reduction of insulin levels and HOMA-IR index that are compatible with an early improvement of insulin sensitivity. We cannot however exclude or quantify the possible contribution of physical activity to this issue.

We also examined cytokines and adipokines for their link to the pathophysiology of obesity and insulin resistance. It is well known that the adipose tissue is an endocrine organ with a wide, biologically active secretome $[38,39]$ and that the adipose tissue of obese individuals is characterized by an increased expression and/or secretion of several proinflammatory cytokines, as well as of adipokines known to promote inflammation, atherogenesis, and insulin 
resistance, whereas the biosynthesis of anti-inflammatory, antiatherogenic, and insulin-sensitising adipokines, such as adiponectin, is decreased [40-42].

Several studies have shown that increased circulating leptin levels and increased adiposity are associated with leptin resistance and that this condition can contribute to the onset and/or maintenance of obesity [43-46]. In our study, we observed, throughout the follow-up, a significant decrease in circulating levels of leptin, which showed a statistically significant positive correlation with both BMI and insulin. Although insulin and leptin resistance have been widely described in the obese subjects, the relationships between these two hormones are complex and not fully understood, and evidence exists that they are associated with body fat through different mechanisms $[47,48]$. No statistical variations of other adipokines or cytokines have been observed after dietary treatment in the whole time period of the study. Other studies with comparable or even greater sample size had obtained similar results and emphasized a statistically significant decrement in leptin levels following diet [49-51]. Leptin, but not adiponectin, resistin, RBP-4, or cytokines, has been correlated with calorie restriction and weight loss in a recent study [52], while an improvement in adipokine profile was observed in severely obese women, only after a 5 to $10 \%$ weight loss [37].

Based on previous findings and on the consideration that $\mathrm{BMI}$ is a good predictor of cardiovascular disease risk $[53,54]$, we hypothesized that weight loss could be associated with an improvement in hemostatic parameters. Due to the small sample size, our results showed nonstatistically significant differences, as also reported by other works that investigated an even greater number of patients $[55,56]$.

An intriguing and apparently equivocal finding in our study is the precocious increase of circulating EGF levels after diet introduction, while EGF decreases in the patient group that had lost $>5 \%$ of body weight, in association with an improvement in insulin sensitivity. Salivary glands are the major source of circulating EGF, a growth factor implicated in the control of cell proliferation and differentiation [57]. During fasting, salivary and plasma EGF physiologically increase [58] to inhibit gastric acid secretion and to preserve the oroesophageal and gastrointestinal mucosal integrity. We hypothesize that the increase of EGF observed in our study is coherent with the food restriction state and with the intestinal adaptation to diet. The increase in EGF may exert insulin-like biological activities in tissues expressing high levels of EGF receptors (EGFR), such as fat and skeletal muscle [59]. By binding to EGFR, a receptor belonging to the tyrosine kinase receptor family, like the insulin receptor [60], EGF can amplify the downstream signaling of insulin through the recruitment of additional PI3-kinase pools in tissues, in which both cognate receptors and the downstream signaling molecules are all abundantly expressed, leading to an EGF-induced translocation of GLUT4 on the plasma membranes and the stimulation of glucose uptake in target tissues [59]. These molecular mechanisms are particularly important in insulin-resistant states, including obesity, in which the increase of EGF due to food restriction may trigger insulin-like compensatory mechanisms. It is also known that insulin regulates EGF expression [61]. This explains the parallel decrease of both insulin and EGF in patients who achieved a more consistent weight loss, in which insulin sensitivity has been improved or reestablished.

$\mathrm{LDH}$ is an intracellular enzyme that is released into the bloodstream when tissues are destroyed or injured. Therefore, $\mathrm{LDH}$ is considered an important clinical marker of tissue or cell integrity. In the obese, the adipose tissue is dysfunctional $[62,63]$ and exhibits a reduced capacity to store and retain nonesterified fatty acids (NEFA), leading to an increase in NEFA circulating levels, thereby promoting the development of lipotoxicity in peripheral tissues [64]. In this study, we observed a significant reduction of circulating levels of $\mathrm{LDH}$ following diet, a condition compatible with an attenuation of the dysfunctional, proinflammatory mechanisms that characterizes obesity.

\section{Conclusions}

On the whole, our study confirms the efficacy of a moderate hypocaloric Mediterranean diet to improve insulin sensitivity in obese patients. The novelty of our findings is that calorie restriction determines a precocious variation of both insulin and leptin even after achieving a modest weight loss. In addition to these traditional laboratory parameters, LDH and EGF have emerged as novel obesity-related markers, pathophysiologically linked to obesity and insulin resistance, that deserve further attention. In particular, the precocious increment of EGF might be clinically useful as an early predictor for a positive evolution of the metabolic response following calorie restriction.

\section{Conflict of Interests}

The authors declare that there is no conflict of interests regarding the publication of this paper.

\section{Acknowledgments}

The authors are indebted to Professor Giuseppe Parlato, Clinical Chemistry Operative Unit of the University of Catanzaro, for the measurement of serum insulin. They also wish to thank the staff of Clinical Pathology, Clinical Biochemistry, and Clinical Nutrition Operative Units of the University of Catanzaro for their help in this study.

\section{References}

[1] World Health Organization, "Obesity: preventing and managing the global epidemic," WHO Technical Report 894, World Health Organization, Geneva. Switzerland, 2000.

[2] C. L. Ogden, M. M. Lamb, M. D. Carroll, and K. M. Flegal, "Prevalence of Obesity in the United States, 2009-2010," NCHS Data Brief, vol. 82, pp. 1-8, 2012.

[3] C. L. Ogden, M. D. Carroll, L. R. Curtin, M. M. Lamb, and K. M. Flegal, "Prevalence of high body mass index in US children and adolescents, 2007-2008," Journal of the American Medical Association, vol. 303, no. 3, pp. 242-249, 2010. 
[4] R. S. Vasan, M. J. Pencina, M. Cobain, M. S. Freiberg, and R. B. D'Agostino, "Estimated risks for developing obesity in the Framingham Heart Study," Annals of Internal Medicine, vol. 143, no. 7, pp. 473-I12, 2005.

[5] S. Klein, L. E. Burke, G. A. Bray et al., "Clinical implications of obesity with specific focus on cardiovascular disease: a statement for professionals from the American Heart Association Council on Nutrition, Physical Activity, and Metabolism," Circulation, vol. 110, no. 18, pp. 2952-2967, 2004.

[6] P. Poirier and R. H. Eckel, "Obesity and cardiovascular disease," Current Atherosclerosis Reports, vol. 4, no. 6, pp. 448-453, 2002.

[7] P. Zimmet and K. G. M. M. Alberti, "Global and societal implications of the diabetes epidemic," Nature, vol. 414, no. 6865, pp. 782-787, 2001.

[8] P. Hossain, B. Kawar, and M. El Nahas, "Obesity and diabetes in the developing world-a growing challenge," The New England Journal of Medicine, vol. 356, no. 3, pp. 213-215, 2007.

[9] G. A. Bray, A Guide to Obesity and the Metabolic Syndrome, CRC Press, 2011.

[10] H. C. Masouka and N. Chalasani, "Nonalcoholic fatty liver disease: an emerging threat to obese and diabetic individuals," Annals of the New York Academy of Sciences, vol. 1281, pp. 106122, 2013.

[11] S. D. Hursting and S. M. Dunlap, "Obesity, metabolic dysregulation, and cancer: a growing concern ad an inflammatory (and microenvironmental) issue," Annals of the New York Academy of Sciences, vol. 1271, pp. 82-87, 2012.

[12] I. Vucenik and J. P. Stains, "Obesity and cancer risk: evidence, mechanisms, and recommendations," Annals of the New York Academy of Sciences, vol. 1271, pp. 37-43, 2012.

[13] T. Gharibeh and R. Mehra, "Obstructive sleep apnea syndrome: natural history, diagnosis, and emerging treatment options," Nature and Science of Sleep, vol. 2, pp. 233-255, 2010.

[14] F. Berenbaum, F. Eymard, and X. Houard, "Osteoarthritis, inflammation an obesity," Current Opinion in Rheumatology, vol. 25, pp. 114-118, 2013.

[15] C. S. Farah and C. M. Salome, "Asthma and obesity: a known association but unknown mechanism," Respirology, vol. 17, no. 3, pp. 412-421, 2012.

[16] D. W. Haslam and W. P. T. James, “Obesity," The Lancet, vol. 366, no. 9492, pp. 1197-1209, 2005.

[17] D. Withrow and D. A. Alter, "The economic burden of obesity worldwide: a systematic review of the direct costs of obesity," Obesity Reviews, vol. 12, no. 2, pp. 131-141, 2011.

[18] M. I. McCarthy, "Genomics, type 2 diabetes, and obesity," The New England Journal of Medicine, vol. 363, no. 24, pp. 23392350, 2010.

[19] J.-P. Després and I. Lemieux, "Abdominal obesity and metabolic syndrome," Nature, vol. 444, no. 7121, pp. 881-887, 2006.

[20] A. Tchernof and J. P. Després, "Pathophysiology of human visceral obesity: an update," Physiological Reviews, vol. 93, pp. 359-404, 2013.

[21] G. A. Rosito, R. B. D’Agostino, J. Massaro et al., "Association between obesity and a prothrombotic state: The Framingham Offspring Study," Journal of Thrombosis and Haemostasis, vol. 91, pp. 638-639, 2004.

[22] C. Bachmayer, A. Lammert, T. Hasenberg, and H. P. Hammes, "Healthy obese and post-bariatric patients-metabolic and vascular patterns," Experimental and Clinical Endocrinology \& Diabetes, vol. 121, no. 8, pp. 483-487, 2013.
[23] G. A. Bray and D. H. Ryan, "Medical therapy for the patient with obesity," Circulation, vol. 125, no. 13, pp. 1695-1703, 2012.

[24] R. H. Eckel, "Nonsurgical management of obesity in adults," The New England Journal of Medicine, vol. 358, no. 18, pp. 1941-1950, 2008.

[25] G. Blackburn, "Effect of degreee of weight loss on health benefits," Obesity Research, vol. 3, supplement 2, pp. 211-216, 1995.

[26] D. J. Goldstein, "Beneficial health effects of modest weight loss," International Journal of Obesity, vol. 16, no. 6, pp. 397-415, 1992.

[27] I. Shai, D. Schwarzfuchs, Y. Henkin et al., "Weight loss with a low-carbohydrate, Mediterranean, or low-fat diet," The New England Journal of Medicine, vol. 359, no. 3, pp. 229-241, 2008.

[28] T. M. Wallace, J. C. Levy, and D. R. Matthews, "Use and abuse of HOMA modeling," Diabetes Care, vol. 27, no. 6, pp. 1487-1495, 2004.

[29] V. Hainer, H. Toplak, and A. Mitrakou, "Treatment modalities of obesity: what fits whom?” Diabetes Care, vol. 31, no. 2, pp. S269-S277, 2008.

[30] E. Heggen, T. O. Klemsdal, F. Haugen, I. Holme, and S. Tonstad, "Effect of a low-fat versus a low-gycemic-load diet on inflammatory biomarker and adipokine concentrations," Metabolic Syndrome and Related Disorders, vol. 10, no. 6, pp. 437-442, 2012.

[31] G. S. Hotamisligil, "Inflammation and metabolic disorders," Nature, vol. 444, no. 7121, pp. 860-867, 2006.

[32] N. Ouchi, J. L. Parker, J. J. Lugus, and K. Walsh, "Adipokines in inflammation and metabolic disease," Nature Reviews Immunology, vol. 11, no. 2, pp. 85-97, 2011.

[33] S. E. Shoelson, J. Lee, and A. B. Goldfine, "Inflammation and insulin resistance," Journal of Clinical Investigation, vol. 116, no. 7, pp. 1793-1801, 2006.

[34] A. C. Fernandez, A. V. Casariego, I. C. Rodreguez, and M. D. B. Pomar, "One-year effectiveness of two hypocaloric diets with different protein/carbohydrate ratios in weight loss and insulin resistance," Nutricion Hospitalaria, vol. 27, no. 6, pp. 2093-2101, 2012.

[35] L. Stern, N. Iqbal, P. Seshadri et al., "The effects of lowcarbohydrate versus conventional weight loss diets in severely obese adults: one-year follow-up of a randomized trial," Annals of Internal Medicine, vol. 140, no. 10, pp. 778-785, 2004.

[36] K. Esposito, R. Marfella, M. Ciotola et al., "Effect of a Mediterranean-style diet on endothelial dysfunction and markers of vascular inflammation in the metabolic syndrome: a randomized trial," Journal of the American Medical Association, vol. 292, no. 12, pp. 1440-1446, 2004.

[37] K. A. Varady, L. Tussing, S. Bhutani, and C. L. Braunschweig, "Degree of weight loss required to improve adipokine concentrations and decrease fat cell size in severely obese women," Metabolism: Clinical and Experimental, vol. 58, no. 8, pp. 10961101, 2009.

[38] Y. Deng and P. E. Scherer, "Adipokines as novel biomarkers and regulators of the metabolic syndrome," Annals of the New York Academy of Sciences, vol. 1212, pp. E1-E19, 2010.

[39] E. E. Kershaw and J. S. Flier, "Adipose tissue as an endocrine organ," Journal of Clinical Endocrinology and Metabolism, vol. 89, no. 6, pp. 2548-2556, 2004.

[40] S. Galic, J. S. Oakhill, and G. R. Steinberg, "Adipose tissue as an endocrine organ," Molecular and Cellular Endocrinology, vol. 316, no. 2, pp. 129-139, 2010. 
[41] R. Rea and R. Donnelly, "Resistin: an adipocyte-derived hormone. Has it a role in diabetes and obesity," Diabetes, Obesity and Metabolism, vol. 6, no. 3, pp. 163-170, 2004.

[42] Y. Arita, S. Kihara, N. Ouchi et al., "Paradoxical decrease of an adipose-specific protein, adiponectin, in obesity," Biochemical and Biophysical Research Communications, vol. 257, no. 1, pp. 79-83, 1999.

[43] J. Wauman and J. Tavernier, "Leptin receptor signaling: pathways to leptin resistance," Frontiers in Bioscience, vol. 16, no. 7, pp. 2771-2793, 2011.

[44] E. C. Villanueva and M. G. Myers Jr., "Leptin receptor signaling and the regulation of mammalian physiology," International Journal of Obesity, vol. 32, no. 7, pp. S8-S12, 2008.

[45] E. Jéquier, "Leptin signaling, adiposity, and energy balance," Annals of the New York Academy of Sciences, vol. 967, pp. 379388, 2002.

[46] J. R. Vasselli, P. J. Scarpace, R. B. Harris, and W. A. Banks, "Dietary components in the development of leptin resistance," Advances in Nutrition, vol. 4, no. 2, pp. 164-175, 2013.

[47] S. Dagogo-Jack, C. Fanelli, D. Paramore, J. Brothers, and M. Landt, "Plasma leptin and insulin relationships in obese and nonobese humans," Diabetes, vol. 45, no. 5, pp. 695-698, 1996.

[48] M. W. Schwartz, R. L. Prigeon, S. E. Kahn et al., "Evidence that plasma leptin and insulin levels are associated with body adiposity via different mechanisms," Diabetes Care, vol. 20, no. 9, pp. 1476-1481, 1997.

[49] K. Anderlová, J. Křemen, R. Doležalová et al., “The influence of very-low-calorie diet on serum leptin, soluble leptin receptor, adiponectin and resistin levels in obese women," Physiological Research, vol. 55, no. 3, pp. 277-283, 2006.

[50] G. Mazzali, V. Di Francesco, E. Zoico et al., "Interrelations between fat distribution, muscle lipid content, adipocytokines, and insulin resistance: Effect of moderate weight loss in older women," The American Journal of Clinical Nutrition, vol. 84, no. 5, pp. 1193-1199, 2006.

[51] E. Klimcakova, M. Kovacikova, V. Stich, and D. Langin, "Adipokines and dietary interventions in human obesity," Obesity Reviews, vol. 11, no. 6, pp. 446-456, 2010.

[52] M. C. Klempel and K. A. Varady, "Reliability of leptin, but not adiponectin, as a biomarker for diet-induced weight loss in humans," Nutrition Reviews, vol. 69, no. 3, pp. 145-154, 2011.

[53] A. Berrington de Gonzalez, P. Hartge, J. R. Cerhan et al., "Bodymass index and mortality among 1.46 million white adults," The New England Journal of Medicine, vol. 363, pp. 2211-2219, 2010.

[54] K. M. Flegal, B. K. Kit, H. Orpana, and B. I. Graubard, "Association of all-cause mortality with overweight and obesity using standard body mass index categories: a systematic review and meta-analysis," Journal of the American Medical Association, vol. 309, no. 1, pp. 71-82, 2013.

[55] A. R. Folsom, H. T. Qamhieh, R. R. Wing et al., "Impact of weight loss on plasminogen activator inhibitor (PAI-1), factor VII, and other hemostatic factors in moderately overweight adults," Arteriosclerosis and Thrombosis, vol. 13, no. 2, pp. 162169, 1993.

[56] I. Mertens and L. F. Van Gaal, "Obesity, haemostasis and the fibrinolytic system," Obesity Reviews, vol. 3, no. 2, pp. 85-101, 2002.

[57] G. Carpenter and S. Cohen, "Epidermal growth factor," The Journal of Biological Chemistry, vol. 265, no. 14, pp. 7709-7712, 1990.
[58] M. Grau, C. Rodríguez, M. Soley, and I. Ramírez, "Relationship between epidermal growth factor in mouse submandibular glands, plasma, and bile: effects of catecholamines and fasting," Endocrinology, vol. 135, no. 5, pp. 1854-1862, 1994.

[59] S. Gogg and U. Smith, "Epidermal growth factor and transforming growth factor $\alpha$ mimic the effects of insulin in human fat cells and augment downstream signaling in insulin resistance," Journal of Biological Chemistry, vol. 277, no. 39, pp. 3604536051, 2002.

[60] J. Schlessinger and A. Ullrich, "Growth factor signaling by receptor tyrosine kinases," Neuron, vol. 9, no. 3, pp. 383-391, 1992.

[61] S. Kasayama, Y. Ohba, and T. Oka, "Epidermal growth factor deficiency associated with diabetes mellitus," Proceedings of the National Academy of Sciences of the United States of America, vol. 86, no. 19, pp. 7644-7648, 1989.

[62] K. J. Strissel, Z. Stancheva, H. Miyoshi et al., "Adipocyte death, adipose tissue remodeling, and obesity complications," Diabetes, vol. 56, no. 12, pp. 2910-2918, 2007.

[63] T. Skurk, C. Alberti-Huber, C. Herder, and H. Hauner, "Relationship between adipocyte size and adipokine expression and secretion," Journal of Clinical Endocrinology and Metabolism, vol. 92, no. 3, pp. 1023-1033, 2007.

[64] C. Lelliott and A. J. Vidal-Puig, "Lipotoxicity, an imbalance between lipogenesis de novo and fatty acid oxidation," International Journal of Obesity, vol. 28, no. 4, pp. S22-S28, 2004. 


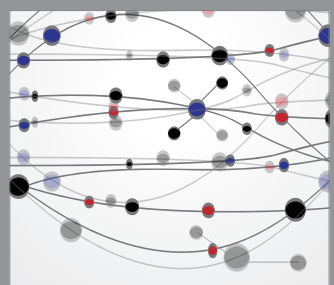

The Scientific World Journal
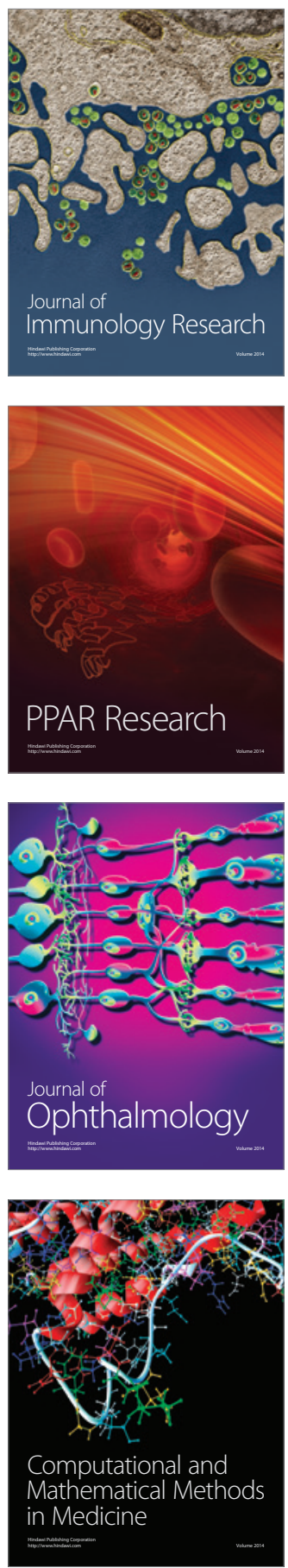

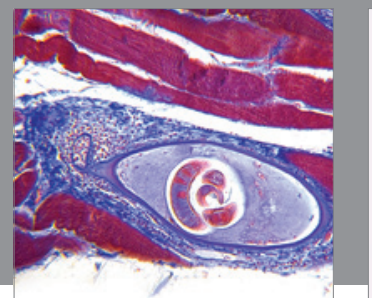

Gastroenterology

Research and Practice
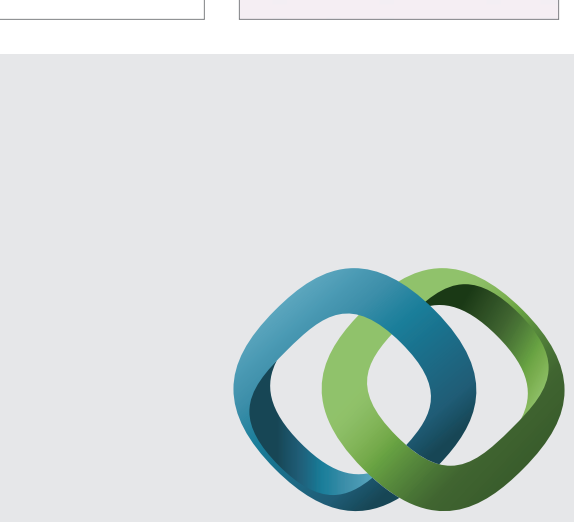

\section{Hindawi}

Submit your manuscripts at

http://www.hindawi.com
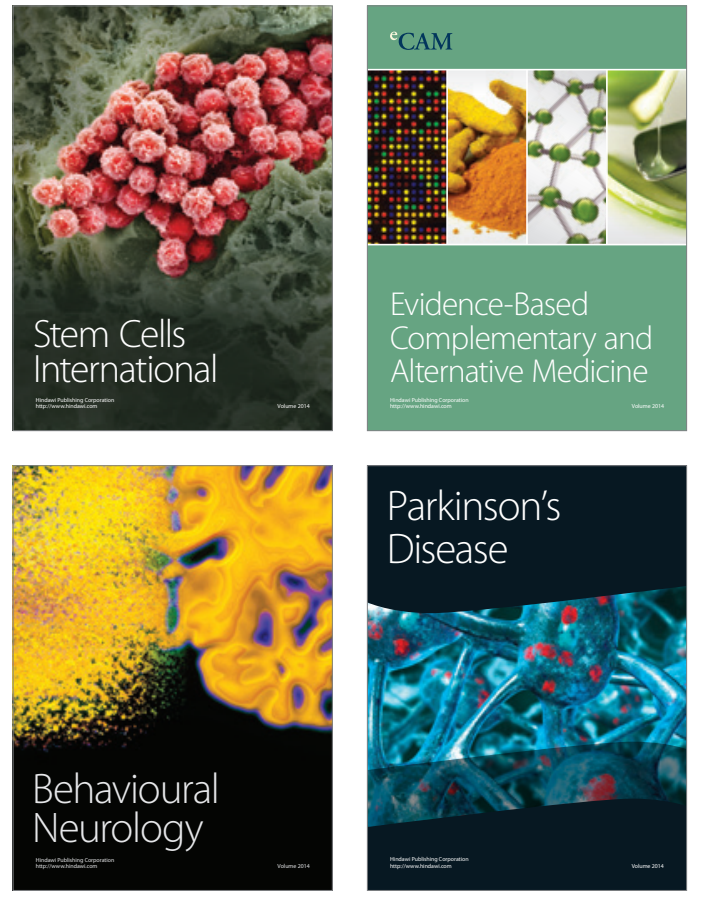
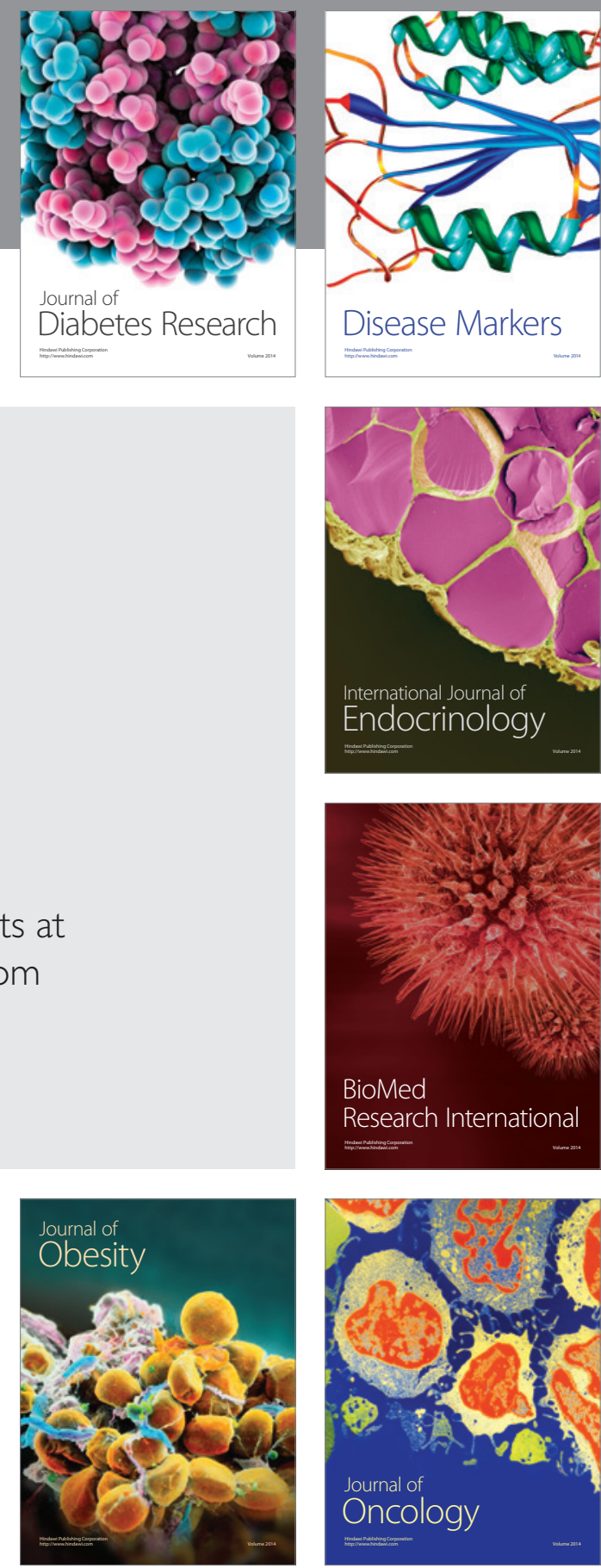

Disease Markers
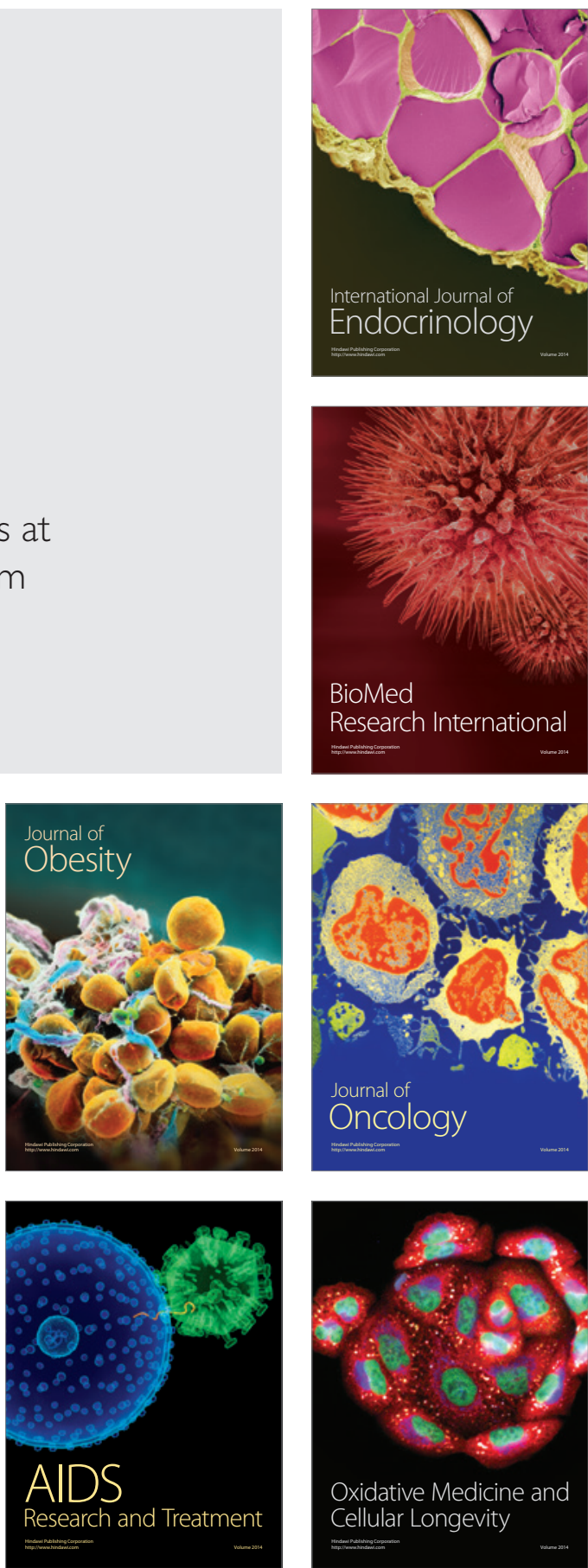